\title{
Spontaneous Rupture of Urinary Bladder in a Young Alcoholic Male
}

\author{
Ritika Zijoo ${ }^{\mathrm{a}}$, Ahmed Dirweesh ${ }^{\mathrm{a}, \mathrm{b}}$, Fausto M. Ordonez ${ }^{\mathrm{a}}$, Anand Kaji ${ }^{\mathrm{a}}$
}

\begin{abstract}
Spontaneous rupture of the urinary bladder (SRUB) is a rare clinical condition. Prompt diagnosis is often difficult both clinically and radiologically and necessitates a high index of suspicion as the patients present with non-specific abdominal pain and may not offer a clear history. We report this young male who presented with SRUB which occurred after alcohol intoxication and presented to us with vague abdominal pain, anuria, and intraperitoneal fluid collection. He had presented with a painful urinary retention few days prior to this visit, was catheterized temporarily and was discharged on antibiotics with a diagnosis of prostatitis. Currently, he stated that he had not urinated since the recent discharge, and noticed increasing abdominal distension. He also had diffuse severe abdominal pain. Patient stated that prior to this, he had consumed 12 beers. Examination revealed a male in acute distress, with normal ranges vital signs. Abdomen was soft, diffusely tender, with sluggish bowel-sounds, and no rebound-tenderness. Rest of examination was normal. Blood workup was significant for elevated BUN and creatinine from his baseline, and leukocytosis. A Foley catheter was placed. His abdomen computed tomography (CT) scan showed a newly developed pneumoperitoneum. CT cysto-urethrogram confirmed intraperitoneal bladder rupture. The patient had an exploratory laparotomy. The bladder defect was closed, and an indwelling catheter was left to ease healing. He had an uneventful postoperative course, and was discharged with a follow-up appointment in the urology clinic. Spontaneous urinary-bladder rupture is a rare event. Excessive alcohol consumption causes diuresis, over-distension and rupture of the urinary bladder. Initial presentation remains imprecise; continuous urinary leakage leads to abscesses, sepsis, and metabolic derangements. CT scan is imaging modality of choice. The condition is a surgical emergency, and necessitates laparotomy and closure of the breach.
\end{abstract}

Keywords: Urinary bladder; Rupture; Alcohol

Manuscript accepted for publication March 24, 2016

aDepartment of Internal Medicine, Seton Hall University, Saint Francis Medical Center, Trenton, NJ, USA

${ }^{\mathrm{b} C o r r e s p o n d i n g ~ A u t h o r: ~ A h m e d ~ D i r w e e s h, ~ S e t o n ~ H a l l ~ U n i v e r s i t y, ~ S a i n t ~ F r a n c i s ~}$ Medical Center, Trenton, NJ, USA. Email: adirweesh@stfrancismedical.org

doi: http://dx.doi.org/10.14740/jmc2470w

\section{Introduction}

Spontaneous rupture of the urinary bladder (SRUB) is a rare clinical condition, with very high morbidity and mortality rates. Idiopathic rupture is encountered in less than $1 \%$ of all cases. This condition is often difficult to diagnose clinically, even with increased timely access to computed tomography (CT). The approach requires a high index of suspicion as the patients present with non-specific abdominal pain and may not provide a clear history [1]. Sometimes the idiopathic spontaneous rupture of the bladder is not even diagnosed or suspected during the victim's life, being revealed during the autopsy.

We report this case of a young patient who presented with SRUB which occurred after alcohol intoxication and presented to us with vague abdominal pain, anuria, and intraperitoneal fluid collection.

\section{Case Report}

A 31-year-old Hispanic male with a remote history of lumbar-spine surgery presented to ER with generalized abdominal pain. He had presented to the ER with a painful urinary retention 4 days prior to this visit and was diagnosed with prostatitis. He was subsequently catheterized and discharged on finasteride and bactrim at that time. In his current visit, he stated that he had not urinated since the prior discharge, and noticed significant abdominal distension. On straining, patient was able to pass drops of blood. He also had diffuse severe abdominal pain, with no alleviating or aggravating factors. Patient stated that he had consumed 12 beers over a course of 12 $\mathrm{h}$ prior to his arrival.

Physical examination revealed a young male in an acute distress. Vitals were noted to be temperature of $98.1^{\circ} \mathrm{F}$, respiratory rate of $16 \mathrm{breath} / \mathrm{min}$, heart rate of 99 beats $/ \mathrm{min}$, and a blood pressure of 142/87 mm Hg. Cardiopulmonary examination did not show any abnormalities. Abdominal examination revealed sluggish bowel sounds, with no visible scars; abdomen was soft, diffusely tender to palpation, with no reboundtenderness or organomegaly. Rest of the physical examination was within normal limits.

Laboratory findings revealed BUN of $43 \mathrm{mg} / \mathrm{dL}$ and creatinine of $5.56 \mathrm{mg} / \mathrm{dL}$, both of which were significantly elevated from patients' baseline. The complete blood count showed 


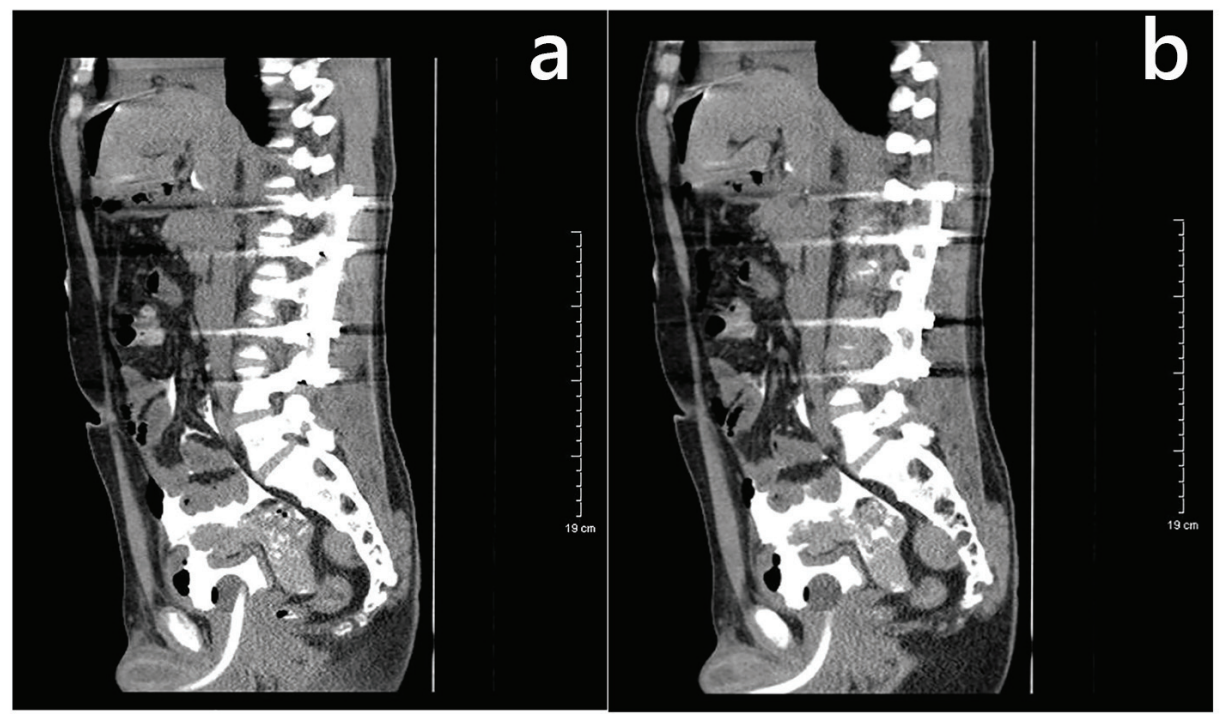

Figure 1. (a, b) CT cystogram showing an intraperitoneal bladder rupture with extensive extravasation of contrast. The defect is seen at the anterior bladder dome.

WBCs of $15.7 \times 10^{3} / \mu \mathrm{L}$ with normal hemoglobin/hematocrit and platelets. The coagulation panel was also normal.

In the emergency department, a three-way Foley catheter was placed draining sanguineous urine. A CT scan of abdomen was ordered and in comparison to the prior done on the patients' previous ER visit, showed a newly developed pneumoperitoneum. The On-Call Urologist was immediately contacted and a CT cysto-urethrogram was performed, which confirmed intraperitoneal bladder rupture at the anterior bladder dome (Fig. 1a, b).

The patient was then immediately taken to the OR for an exploratory laparotomy. The bladder defect was closed in two layers (water-tight closure) using a continuous technique. An indwelling catheter was left to ease healing of the bladder repair. The patient had an uneventful postoperative. He was discharged home with a fixed follow-up in the out-patient urology clinic.

\section{Discussion}

SRUB is a rare event. The clinical presentation is usually with features of peritonitis and most of the time, the diagnosis is made intraoperatively. The condition carries high rates of morbidities and mortalities (47\%) [2], attributed to the delay in diagnosis. It is often difficult to distinguish SRUB from other causes of acute abdomen especially in the absence of a history of traumatic event [3].

The most common causes of atraumatic rupture of urinary bladder are chronic inflammation, bladder outflow obstruction (BOO) urothelial tumors, and radiotherapy. Intra-peritoneal ruptures are divided in two categories, depending on their etiology: rupture of the bladder secondary to a pathological lesion (chronic infections, tuberculosis, eosinophilic or necrotic cystitis, pelvic irradiation for cervical cancer, urothelial tumors) and rupture by over-distension. The spontaneous rupture of the bladder can be facilitated by excessive alcohol consumption which has a diuretic effect [4] and leads to overdistension in the context of the sensitivity disturbances due to the $\alpha$-sympathomimetic effect and CNS depression produced by the alcohol. In men, congestion of the prostate and prostatic urethra aggravates the obstruction of the outlet [5]. With extreme increase of the intra-vesical pressure, the most susceptible part is the intra-peritoneal bladder [6].

In the literature, an accurate preoperative diagnosis of urinary bladder rupture was made only in few cases $[7,8]$, and many of these cases were initially treated conservatively with urinary catheterization and antibiotics.

During the initial stages, the clinical manifestations of bladder rupture remain imprecise, and diffuse including pain and difficulty of urinating, and after the rupture, the signs and symptoms of peritonitis and urinary difficulties can be detected [9]. Complications caused by the leakage of the urine into the peritoneal cavity and pelvis include intra-abdominal and pelvic abscesses, sepsis, and metabolic derangements. Significant peritoneal reabsorption of urea and creatinine can masquerade as acute renal failure on initial biochemical testing, as observed in our patient. Electrolyte abnormalities such as hyperkalemia, hypernatremia, uremia, or acidosis may also occur as extravasated urine is reabsorbed in the peritoneal cavity. Electrocardiogram abnormalities resulting from hyperkalemia may be seen. Several authors have noted increased mortality rates after severe symptom onset $[10,11]$.

Imaging modalities to evaluate a patient with suspected bladder injury have included retrograde cystography, CT retrograde cystography, and delayed CT cystography. Conventional abdominal non-contrast CT has an accuracy rate of only $60.6 \%$ for bladder injury compared to $95.9 \%$ for the retrograde cystogram [12]. However, in a patient with worsening abdominal pain/distention and vital sign abnormalities, a CT scan will likely be the imaging modality of choice.

SRUB is a surgical emergency and can be rapidly fatal if 
diagnosis and treatment are delayed, with early and adequate treatment being essential $[6,13]$. The treatment consists of laparotomy and suture as continuous leakage of urine does not allow spontaneous closure of the breach [6].

\section{Conclusion}

Most cases of spontaneous bladder rupture in relation to excessive alcohol consumption can have a positive outcome with early prediction and management. This diagnosis should be considered in those who present with acute abdomen, anuria and a raised serum creatinine level. Although the idiopathic SRUB is a rarely encountered, all medical professionals working in ER settings need to be aware of this presentation. Prompt surgical consultation and management would prevent a poor clinical outcome in such cases.

\section{Grant Support}

None.

\section{Conflicts of Interest}

None.

\section{References}

1. Daignault MC, Saul T, Lewiss RE. Bedside ultrasound diagnosis of atraumatic bladder rupture in an alcoholintoxicated patient: a case report. Crit Ultrasound J. 2012;4(1):9.

2. Hansen HJ, Eldrup J. Spontaneous rupture of the urinary bladder - a late complication to radiotherapy. Case report.
Scand J Urol Nephrol. 1989;23(4):309-310.

3. Muneer M, Abdelrahman H, El-Menyar A, Zarour A, Awad A, Al-Thani H. Spontaneous Atraumatic Urinary Bladder Rupture Secondary to Alcohol Intoxication: A Case Report and Review of Literature. Am J Case Rep. 2015;16:778-781.

4. Bastable JR, De Jode LR, Warren RP. Spontaneous rupture of the bladder. Br J Urol. 1959;31(1):78-86.

5. Kim HJ, Moon H, Sohng I, Lee HW, Lee G, Lee JG. Effects of ethanol and its metabolite acetaldehyde on responses of the rat bladder. BJU Int. 1999;83(6):686-692.

6. Jurisic D, Glavan E, Cugura JF, Vidovic D. Splenic rupture following idiopathic rupture of the urinary bladder presenting as acute abdomen. J Postgrad Med. 2007;53(1):39-40.

7. Atalay AC, Karaman MI. Spontaneous rupture of a bladder with invasive bladder carcinoma. Int Urol Nephrol. 1998;30(6):723-724.

8. Wujanto R, Brough R, O'Reilly PH. Spontaneous perforation of squamous cell bladder carcinoma associated with hypercalcaemia. Br J Urol. 1989;63(6):647-648.

9. Faraj R, O'Donovan P, Jones A, Hill S. Spontaneous rupture of urinary bladder in second trimester of pregnacy. Internet Journal of Gynecology \& Obstetrics. 2009;10(2):2.

10. Sezhian N, Rimal D, Suresh G. Isolated intraperitoneal bladder rupture following minor trauma after alcohol ingestion. South Med J. 2005;98(5):573-574.

11. Herd AM, Crofts NG, Lee LM, Girard JV, Sweetland RJ. Isolated bladder rupture after minor trauma in a patient with alcohol intoxication. J Emerg Med. 1994;12(3):409411.

12. Hsieh CH, Chen RJ, Fang JF, Lin BC, Hsu YP, Kao JL, Kao YC, et al. Diagnosis and management of bladder injury by trauma surgeons. Am J Surg. 2002;184(2):143147.

13. Arnaout F. Spontaneous atraumatic rupture of urinary bladder. Internet Journal of Urology. 2008;5(2). 